\title{
761.
}

\section{ON THE THEOREM OF THE FINITE NUMBER OF THE COVARIANTS OF A BINARY QUANTIC.}

[From the Quarterly Journal of Pure and Applied Mathematics, vol. xvII. (1881), pp. 137-147.]

GoRDAN's proof, the only one hitherto given, is based upon the theory of derivatives (Uebereinanderschiebungen). It is shown that the irreducible covariants of the binary quantic $f$ are included in the series

$$
(f, f)^{2},(f, f)^{4}, \ldots(f, h),(f, h)^{2}, \ldots
$$

of the derivatives of the quantic upon itself or upon some other covariant, and that the number of the irreducible covariants thus obtained is finite. And not only so, but for the quintic and the sextic the complete systems were formed, and the numbers shown to be $=23$ and 26 respectively.

It would seem that there ought to be a more simple proof based upon the consideration of the fundamental covariants: for the cubic $(a, b, c, d \gamma x, y)^{3}$, these are the cubic itself $(a, \ldots \gamma x, y)^{3}$, the Hessian $\left(a c-b^{2}, \ldots \gamma x, y\right)^{2}$, and the cubicovariant $\left(a^{2} d-3 a b c+2 b^{3}, \ldots \gamma x, y\right)^{3}$; and so in general for the quantic $(a, \ldots \gamma x, y)^{n}$, we have a series of fundamental covariants the leading coefficients whereof are the seminvariants

$$
a, a c-b^{2}, a^{2} d-3 a b c+2 b^{3}, a^{3} e-4 a^{2} b d+6 a b^{2} c-3 b^{4}, \& c .
$$

It is known that every covariant can be expressed as a rational function of these, or more precisely that every covariant multiplied by a positive integral power of the quantic itself can be expressed as a rational and integral function of the fundamental covariants, and we may for the covariants substitute their leading coefficients, or say the seminvariants; hence, every seminvariant is a rational function of the fundamental seminvariants, and more precisely, every seminvariant multiplied by a positive integral 
power of the first coefficient $a$ is a rational function of the fundamental seminvariants. Thus, in the case of the cubic, we have the discriminant $\nabla$,

obtained from

$$
=a^{2} d^{2}-6 a b c d+4 a c^{3}+4 b^{3} d-3 b^{2} c^{2},
$$

by the formula

$$
a, a c-b^{2}, a^{2} d-3 a b c+2 b^{3},
$$

$$
a^{2} \nabla=\left(a^{2} d-3 a b c+2 b^{3}\right)^{2}+4\left(a c-b^{2}\right)^{3},
$$

and it is easily shown that this invariant $\nabla$ is the only new covariant thus obtainable, and that every other covariant is thus a rational and integral function of the irreducible covariants, the leading coefficients of which are

$$
a, a c-b^{2}, a^{2} d-3 a b c+2 b^{3},
$$

and $\nabla$. It appears a truism, and it might be thought that it would be, if not easy, at least practicable, to show for a quantic of any given finite order $n$, that we can in this manner, as rational functions of the $n-1$ seminvariants, obtain only a finite number of new seminvariants, so that all the seminvariants would be expressible as rational and integral functions of a finite number of seminvariants; and, consequently, all the covariants be expressible as rational and integral functions of a finite number of irreducible covariants. But the large number, 23, of the covariants of the quintic is enough to show that the proof, even if it could be carried out, would involve algebraical operations of great complexity.

The theory may be considered from a different point of view, in connexion with the root-form $a(x-\alpha y)(x-\beta y) \ldots$, or say $(x-\alpha)(x-\beta) \ldots$ of the quantic; we have here what may be called the monomial form of covariant, viz. the general monomial form is

$$
(\alpha-\beta)^{m}(\alpha-\gamma)^{n}(\beta-\gamma)^{p} \ldots(x-\alpha)^{q}(x-\beta)^{r} \ldots,
$$

where in all the factors (whether $\alpha-\beta$ or $x-\alpha$ ) which contain $\alpha$, in all the factors which contain $\beta, \ldots$, and so for each root in succession, the sum of the indices has one and the same value, $=\theta$ suppose. Thus, for the cubic

$$
(x-\alpha)(x-\beta)(x-\gamma),
$$

we have the monomial covariants

$$
\begin{gathered}
(\alpha-\beta)(\alpha-\gamma)(\beta-\gamma), \\
(\beta-\gamma)(x-\alpha),(\alpha-\gamma)(x-\beta),(\alpha-\beta)(x-\gamma), \\
(x-\alpha)(x-\beta)(x-\gamma)
\end{gathered}
$$

and so for the quartic

$$
(x-\alpha)(x-\beta)(x-\gamma)(x-\delta),
$$

we have the monomial invariants

$$
(\alpha-\beta)(\gamma-\delta),(\alpha-\gamma)(\beta-\delta),(\alpha-\delta)(\beta-\gamma) .
$$

Observe that the monomial form is considered as essential; a syzygetic function of C. XI. 
two or more monomials is not a monomial, and we are thus in no wise concerned with identities such as

$$
(\beta-\gamma)(x-\alpha)-(\alpha-\gamma)(x-\beta)+(\alpha-\beta)(x-\gamma)=0
$$

or

$$
(\alpha-\delta)(\beta-\gamma)-(\beta-\delta)(\alpha-\gamma)+(\gamma-\delta)(\alpha-\beta)=0
$$

notwithstanding these syzygies respectively,

$$
(\beta-\gamma)(x-\alpha),(\alpha-\gamma)(x-\beta), \text { and }(\alpha-\beta)(x-\gamma)
$$

are regarded as independent covariants of the cubic, and

$$
(\alpha-\delta)(\beta-\gamma),(\beta-\delta)(\alpha-\gamma), \text { and }(\gamma-\delta)(\alpha-\beta),
$$

as independent invariants of the quartic.

It is only when a monomial covariant is equal to a power or product of simple or other powers of lower monomial covariants that it is regarded as a function of these lower monomial covariants and therefore as not irreducible. Thus

$$
(\alpha-\beta)(\alpha-\gamma)(\beta-\delta)(\gamma-\delta)=(\alpha-\beta)(\gamma-\delta) \cdot(\alpha-\gamma)(\beta-\delta)
$$

is a reducible monomial covariant, expressible in terms of the lower irreducible monomial covariants

$$
(\alpha-\beta)(\gamma-\delta) \text { and }(\alpha-\gamma)(\beta-\delta)
$$

The theorem of the finite number of the irreducible monomial covariants (as just explained) of the root-quantic is a question of the same kind as, but entirely distinct from, that of the finite number of the covariants of the quantic in the ordinary form; and there are thus the two questions; $(A)$, that of the finite number of the irreducible monomial covariants of the root-quantic; and $(C)$, that of the finite number of the irreducible covariants of the ordinary quantic.

But we can pass from $(A)$ to $(C)$ by means of a lemma $(B)$, which I have not proved, but which seems highly probable, and which I enunciate as follows: $(B)$ The infinite system of terms $X$, rational and integral functions of a finite set of letters $(a, b, c, \ldots)$ which remain unaltered by all the substitutions of a certain group $G(a, b, c, \ldots)$ of substitutions upon these letters, includes always a finite set of terms $P$ such that every term $X$ whatever is a rational and integral function of these terms $P$.

In explanation of this lemma, observe that, if $G(a, b, c, \ldots)$ denotes the entire group of substitutions upon these letters, so that the functions which remain unaltered by the substitutions of the group are in fact the symmetrical functions of $(a, b, c, \ldots)$, then the theorem is "The infinite system of rational and integral symmetrical functions of $(a, b, c, \ldots)$ includes always a finite set of terms $P$ such that every such rational and integral symmetrical function is a rational and integral function of the terms $P$, viz. the terms $P$ are here the several symmetrical functions

$$
a+b+c+\ldots, a b+a c+b c+\ldots, a b c+\ldots, \& c . "
$$


and so, if $G(\mathrm{a}, \mathrm{b}, \mathrm{c}, \ldots)$ is the group of all the positive substitutions, then we have the like theorem for the rational and integral two-valued functions of $(a, b, c, \ldots)$, viz. the terms $P$ are here the two-valued function $(a-b)(a-c)(b-c) \ldots$, and the symmetrical functions

$$
a+b+c+\ldots, a b+a c+b c+\ldots, a b c+\ldots, \& c .
$$

as before.

I return to the theorem $(A)$, but instead of the covariants of a root-quantic of any order, I consider first the invariants of a root-quantic of any even order. The general form is

$$
(\alpha-\beta)^{m}(\alpha-\gamma)^{n}(\beta-\gamma)^{p} \ldots,
$$

where in all the factors which contain $\alpha$, in all the factors which contain $\beta$, and so for each root in succession, the sum of the indices has one and the same value $=\theta$. Writing 12 for the index of $\alpha-\beta, 13$ for that of $\alpha-\gamma$, and so in other cases, then assuming always $12=21,13=31$, \&c., the indices, taken each twice, form the square

\begin{tabular}{|c|c|c|c|}
\hline 0 & 12 & 13 & $\cdots$ \\
\hline 21 & 0 & 23 & $\cdots$ \\
\hline 31 & 32 & 0 & \\
\hline$\vdots$ & & & \\
\hline & & \\
\hline
\end{tabular}

the order of which, or number of its rows or columns, is equal to the order of the quantic; the terms of the dexter diagonal are each $=0$, and the square is symmetrical in regard to this dexter diagonal. Moreover, the square is such, that the sum of the terms in each row (or column) has one and the same value $=\theta$; and conversely, every such square, say $R_{\theta}$, represents an invariant.

Thus, for the quartic $(x-\alpha)(x-\beta)(x-\gamma)(x-\delta)$, the square $R_{\theta}$ is a square of four rows (or columns) representing the invariant

$$
\begin{array}{r}
(\alpha-\beta)^{12}(\alpha-\gamma)^{13}(\alpha-\delta)^{14} \\
(\beta-\gamma)^{23}(\beta-\delta)^{24} \\
(\gamma-\delta)^{34}
\end{array}
$$

in which

$$
\begin{array}{r}
12+13+14=\theta \\
21+23+24=\theta \\
31+32+34=\theta \\
41+42+43 \quad=\theta
\end{array}
$$


There are three squares $R_{1}$, viz. these are the squares
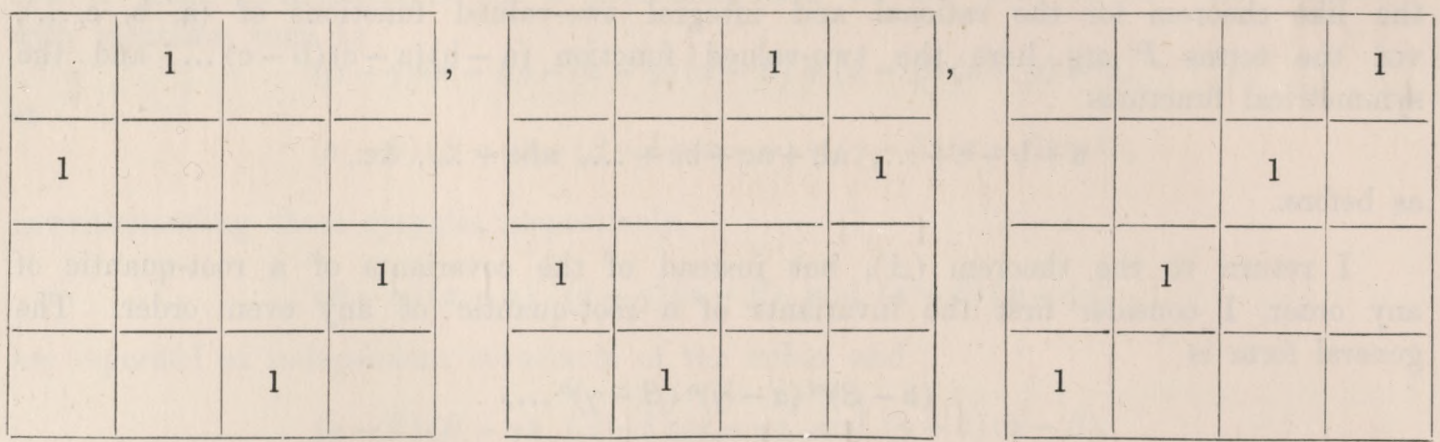

representing the before-mentioned invariants $(\alpha-\beta)(\gamma-\delta),(\alpha-\gamma)(\beta-\delta),(\alpha-\delta)(\beta-\gamma)$ respectively: say these are $\mathrm{a}, \mathrm{b}, \mathrm{c}$, and every other invariant is a rational and integral function of these; in fact, the $\theta$-equations give easily $12=34,13=24,14=23$, so that the general form of the invariant is $=\mathrm{a}^{12} \mathrm{~b}^{13} \mathrm{c}^{14}$, where $12,13,14$ are each of them a positive integer number (which may be $=0$ ). Or, what is the same thing, the square $R_{\theta}(\theta=12+13+14)$ is a sum

$$
=12 \cdot R_{1}+13 \cdot R_{1}^{\prime}+14 \cdot R_{1}^{\prime \prime} \text {, }
$$

with positive integer coefficients $12,13,14$, say for shortness it is a sum of squares $R_{1}$. And so any like expression with a negative coefficient or coefficients may, for shortness, be called a difference of squares $R_{1}$.

Observe that, in general, two squares $R_{\theta}, R_{\phi}$ are added together by adding their corresponding terms, the result being a square $R_{\theta+\phi}$; similarly, if each term of $R_{\phi}$ be less than or at most equal to the corresponding term of $R_{\theta}$, then (but not otherwise) the square $R_{\phi}$ may be subtracted from $R_{\theta}$, giving a square $R_{\theta-\phi}$.

In the case of the sextic

$$
(x-\alpha)(x-\beta)(x-\gamma)(x-\delta)(x-\epsilon)(x-\zeta),
$$

there are fifteen squares $R_{1}$, which may be represented as follows:

\begin{tabular}{l|l}
12.34 .56 & $x_{1}$ \\
12.35 .46 & $y_{1}$ \\
12.36 .45 & $z_{1}$ \\
13.24 .56 & $x_{2}$ \\
13.25 .46 & $y_{2}$ \\
13.26 .45 & $z_{2}$ \\
14.23 .56 & $x_{3}$ \\
14.25 .36 & $y_{3}$ \\
14.26 .35 & $z_{3}$ \\
15.23 .46 & $x_{4}$ \\
15.24 .36 & $y_{4}$ \\
15.26 .34 & $z_{4}$ \\
16.23 .45 & $x_{5}$ \\
16.24 .35 & $y_{5}$ \\
16.25 .34 & $z_{5} ;$
\end{tabular}


viz. 12.34.56 here represents the square $R_{1}$, for which the terms $12,34,56$ (and of course the symmetrical terms $21,43,65)$ are each $=1$, the other terms all vanishing; or, what is the same thing, it represents the invariant $(\alpha-\beta)^{12}(\gamma-\delta)^{34}(\epsilon-\zeta)^{56}$. But it is not true that every square $R_{\theta}$ is a sum of squares $R_{1}$; this is not the case, for the square $R_{2}$,

representing the invariant

$$
=12 \cdot 13 \cdot 23 \cdot 45 \cdot 46 \cdot 56 \text {, }
$$

$$
(\alpha-\beta)^{12}(\alpha-\gamma)^{13}(\beta-\gamma)^{23}(\delta-\epsilon)^{45}(\delta-\zeta)^{46}(\epsilon-\zeta)^{56},
$$

is not a sum of squares $R_{1}$.

But the square last referred to is a difference of squares $R_{1}$ : it is in fact

$$
=12.36 .45+13.25 .46+14.23 .56-14.25 .36 \text {, }
$$

or, what is the same thing, the corresponding invariant is the product of the invariants $12.36 .45,13.25 .46,14.23 .56$, divided by the invariant 14.25 .36 ; viz. it is a rational function of invariants $R_{1}$.

It is required to show, first, that every square $R_{\theta}$ is a difference of squares $R_{1}$; and thence, secondly, that it is a sum of a finite number of squares $R_{k}$ (being, in fact, squares $R_{1}$ and $R_{2}$ ).

For the first theorem we equate the general expression of $R_{\theta}$ with the assumed value

$$
x_{1} \cdot 12.34 .56+y_{1} .12 .35 .46+z_{1} \cdot 12.36 .45+\ldots+z_{5} .16 .25 .34 .
$$

We thus obtain

$$
\begin{aligned}
& \text { fifteen equations } \\
& \text { satisfied by }
\end{aligned}
$$

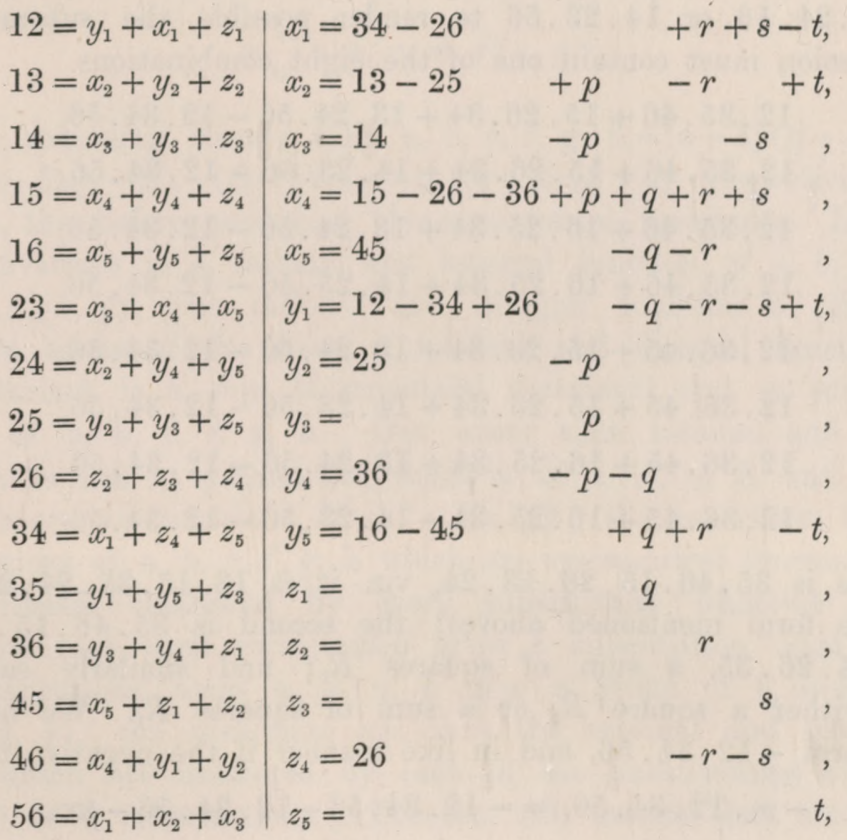


connecting $x_{1}, y_{1}, z_{1}, \ldots, z_{5}$ with the terms 12,13 , etc. of $R_{\theta}$ (or indices of the corresponding invariants). The fifteen equations are not independent, for regarding them as giving the values of $12,13, \ldots$ in terms of the $x_{1}, y_{1}, z_{1}, \ldots, z_{5}$, these values satisfy identically the relations which ought to be satisfied by the terms 12 , 13, etc., viz. the equations obtained by the elimination of $\theta$ from the equations

$$
\begin{array}{r}
12+13+14+15+16=\theta, \\
12 \quad+23+24+25+26=\theta, \\
\vdots \quad 26+36+46+56 \quad=\theta .
\end{array}
$$

The equations are thus insufficient to determine the values of $x_{1}, y_{1}, z_{1}, \ldots, z_{5}$, and the general values given by the equations will contain five indeterminate quantities which are taken to be $p, q, r, s, t$ (these being in fact the values of $y_{3}, z_{1}, z_{2}, z_{3}, z_{5}$ respectively), and we then have the equations all of them satisfied by the above-mentioned values containing these indeterminate quantities; taking them to be positive or negative integers, then $x_{1}, y_{1}, z_{1}, \ldots, z_{5}$, will be all of them integers; but by what precedes, it appears that they cannot all of them be made to be positive integers, so that we have consequently $R_{\theta}$,

$$
=x_{1} \cdot 12 \cdot 34.56+y_{1} \cdot 12 \cdot 35.46+z_{1} \cdot 12 \cdot 36.45+\ldots+z_{5} \cdot 16.25 .34 \text {, }
$$

equal in general to a difference of squares $R_{1}$.

Suppose in such difference of squares $R_{1}$ we have any term, say -12.34 .56 , occurring with the coefficient -1 . Since the expression represents a square $R_{\theta}$, we must have among the positive terms, 12.35 .46 or 12.36 .45 to render possible the subtraction of the $12 ; 15.26 .34$ or 16.25 .34 to render possible the subtraction of the 34 ; and 13.24 .56 or 14.23 .56 to render possible the subtraction of the 56 ; that is, the expression must contain one of the eight combinations

$$
\begin{aligned}
& 12.35 .46+15.26 .34+13.24 .56-12.34 .56, \\
& 12.35 .46+15.26 .34+14.23 .56-12.34 .56, \\
& 12.35 .46+16.25 .34+13.24 .56-12.34 .56, \\
& 12.35 .46+16.25 .34+14.23 .56-12.34 .56, \\
& 12.36 .45+15.26 .34+13.24 .56-12.34 .56, \\
& 12.36 .45+15.26 .34+14.23 .56-12.34 .56, \\
& 12.36 .45+16.25 .34+13.24 .56-12.34 .56, \\
& 12.36 .45+16.25 .34+14.23 .56-12.34 .56 .
\end{aligned}
$$

The first of these is 35.46 .15 .26 .13 .24 , viz. it is 13.15 .35 .24 .26 .46 which is a square $R_{2}$ (of the form mentioned above); the second is 35.46 .15 .26 .14 .23 , which is $15.23 .46+14.26 .35$, a sum of squares $R_{1}$; and similarly each of the other combinations is either a square $R_{2}$ or a sum of squares $R_{1}$. We have thus got rid of the negative term -12.34 .56 , and in like manner if the negative term had been

$$
-m .12 .34 .56,=-12.34 .56-12.34 .56-\& c \text {., }
$$


or, whatever the negative terms may be, we get rid one by one of each negative term; and thus ultimately express $R_{\theta}$ as a sum of squares $R_{1}$ and $R_{2}$. Or, what is the same thing, the invariant $R_{\theta}$ originally expressed as a rational function of invariants $R_{1}$, is finally expressed as a rational and integral function of invariants $R_{1}$ and $R_{2}$.

Similarly for a root-quantic of any even order $n$, we have the general square $R_{\theta}$ expressed, first as a difference of squares $R_{1}$, and then as a sum of squares $R_{1}, R_{2}$, or it may be higher squares $R_{3}$, \&c., but certainly as a sum of a finite number of squares $R_{k}$. For a root-quantic of any odd order $n$, the investigation would be of a somewhat different form, since here there are no squares $R_{1}$, but the lowest squares are squares $R_{2}$ of a form such as 12.23.34.45.15; but the general conclusion would still follow that every square $R_{\theta}$ is a sum of a finite number of squares $R_{k}$. And a like reasoning would apply to covariants instead of invariants: viz. the reasoning (although for simplicity it has been given for a very particular and special case) does, I think, really establish the theorem $(A)$ in its generality, viz. the theorem that for a root-quantic of any given finite order, the number of irreducible monomial covariants is finite.

From any monomial covariant of the root-quantic, by taking the sum of the forms belonging to the different roots, so as to obtain a symmetrical function of the roots, that is, a rational and integral function of the coefficients, we obtain a covariant of the quartic in its ordinary form $(a, \ldots)(x, y)^{n}$. Consider for a moment the beforementioned case of the invariants of the root-quartic

now put

$$
(x-\alpha y)(x-\beta y)(x-\gamma y)(x-\delta y),
$$

$$
=\frac{1}{a}\left(a, b, c, d, e \gamma(x, y)^{4} ;\right.
$$

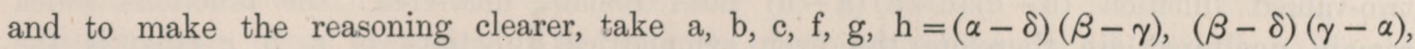
$(\gamma-\delta)(\alpha-\beta),(\alpha-\delta)(\gamma-\beta), \quad(\beta-\delta)(\alpha-\gamma),(\gamma-\delta)(\beta-\alpha)$ respectively, these being, with the signs \pm , the before-mentioned three monomial invariants. In the root-theory, every monomial invariant is a rational and integral function of a, b, c, f, g, h. Every invariant of $(a, \ldots) x, y)^{4}$, quì rational and integral function of the coefficients, is, when expressed in terms of the roots, a rational and integral function of the roots, and then quà invariant is a sum of monomial invariants, and as such a rational and integral function of $a, b, c, f, g, h$. But every such rational and integral function of $\mathrm{a}, \mathrm{b}, \mathrm{c}, \mathrm{f}, \mathrm{g}, \mathrm{h}$ is not a symmetrical function of $\alpha, \beta, \gamma, \delta$, and consequently not in the present theory an invariant of $(a, \ldots)(x, y)^{4}$; the invariants are those rational and integral functions of $\mathrm{a}, \mathrm{b}, \mathrm{c}, \mathrm{f}, \mathrm{g}, \mathrm{h}$ which are symmetrical functions of $(\alpha, \beta, \gamma, \delta)$, that is, which remain unaltered by every substitution whatever upon the roots $(\alpha, \beta, \gamma, \delta)$. Now each such substitution gives a substitution upon a, b, c, f, g, h, and the 24 substitutions upon $\alpha, \beta, \gamma, \delta$ give a group of $6,=\frac{1}{4} .24$ substitutions upon $(\mathrm{a}, \mathrm{b}, \mathrm{c}, \mathrm{f}, \mathrm{g}, \mathrm{h})$; the invariants are thus the rational and integral functions of $(a, b, c, f, g, h)$ which are unaltered by each of the substitutions of a certain group $G(\mathrm{a}, \mathrm{b}, \mathrm{c}, \mathrm{d}, \mathrm{e}, \mathrm{f})$ of 6 substitutions. Theorem $(B)$ asserts that, among the terms in 
question, that is, among such rational and integral functions of $(a, b, c, f, g, h)$, we have a finite number of terms $P$, such that every one of the terms is a rational and integral function of the terms $P$; and recollecting that $a+b+c=0$, these terms $P$ are in fact two terms $b c+c a+a b$ and $(b-c)(c-a)(a-b)$; the conclusion being, that the invariants of the quartic $\left(a, b, c, d, e \gamma(x, y)^{4}\right.$ are all of them rational and integral functions of the last-mentioned two functions, that is, of

$$
I,=a e-4 b d+3 c^{2} \text {, and } J,=a c e-a d^{2}-b^{2} e+2 b c d-c^{3} .
$$

As regards the group $G(\mathrm{a}, \mathrm{b}, \mathrm{c}, \mathrm{f}, \mathrm{g}, \mathrm{h})$ of 6 substitutions upon a, b, c, f, g, h, observe that the 24 substitutions of $(\alpha, \beta, \gamma, \delta)$ operating upon $a, b, c, f, g, h$ give 6 substitutions taken each four times; for instance, the substitutions $1, \alpha \beta \cdot \gamma \delta, \alpha \gamma \cdot \beta \delta$, $\alpha \delta . \beta \gamma$ leave each of them a, b, c, f, g, h unaltered, that is, they each give the substitution 1. And we thus find for the group $G(\mathrm{a}, \mathrm{b}, \mathrm{c}, \mathrm{f}, \mathrm{g}, \mathrm{h})$ the 6 substitutions

$$
\begin{aligned}
& 1, \\
& \text { abc.fgh, } \\
& \text { acb.fhg, } \\
& \text { af . bh.cg, } \\
& \text { ah . bg.cf, } \\
& \text { ag. bf .ch. }
\end{aligned}
$$

For the functions of $a, b, c, f, g, h$, which remain unaltered by the substitution of this group, observe that we have $f, g, h=-a,-b,-c$; so that any function of the six letters may be represented as a functiou of a, b, c. An odd symmetrical function, for instance abc, does not remain unaltered, for it is by any one of the last three substitutions changed into fgh, that is, into - abc; on the other hand, the two-valued function $(b-c)(c-a)(a-b)$ does remain unaltered: the functions which remain unaltered are therefore the even symmetrical functions of a, b, c (that is, the symmetric functions $a^{2}+b^{2}+c^{2}$, or $a b+a c+b c$, \&c., which are of an even order in $a, b, c$ conjointly), and the same even functions multiplied by $(b-c)(c-a)(a-b)$; and having regard to the relation $a+b+c=0$, all these can be expressed as already mentioned as rational and integral functions of $b c+c a+a b$ and $(b-c)(c-a)(a-b)$.

The proof applies to the general case of the theorem $(C)$, viz. taking the theorem (A) to be proved, and putting the root-quantic

$$
(x-\alpha y)(x-\beta y) \ldots=\frac{1}{a}(a, \ldots)(x, y)^{n},
$$

then we have $a, b, c, d, \ldots$ a system of monomial covariants of the root-quantic; and all the covariants of $(a, \ldots \gamma(x, y)$ are rational and integral functions of $(a, b, c, d, \ldots)$ which remain unaltered by the substitutions of a certain group $G(a, b, c, d, \ldots)$; hence, assuming the theorem $(B)$, they are rational and integral functions of a finite number of irreducible covariants. And the demonstration thus depends upon that of the theorem $(B)$. 